\title{
Disambiguation of Polysemous Verbs for Rule-based Inferencing
}

\author{
Fabienne Martin, Dennis Spohr, and Achim Stein \\ Institut für Linguistik/Romanistik \\ Universität Stuttgart, Germany \\ firstname. lastname@ling.uni-stuttgart.de
}

\begin{abstract}
We present an approach to disambiguating verb senses which differ w.r.t. the inferences they allow. It combines standard ontological tools and formalisms with a formal semantic analysis and is hence more formalised and more detailed than existing lexical semantic resources like WordNet and FrameNet [Fellbaum, 1998, Baker et al., 1998]. The resource presented here implements formal semantic descriptions of verbs in the Web Ontology Language (OWL) and exploits its reasoning potential based on Description Logics (DL) for the disambiguation of verbs in context, since before the correct sense of a verb can be reliably determined, its syntactic arguments have to be disambiguated first. We present details on this process, which is based on a mapping from the French EuroWordNet [Vossen, 1998] to SUMO [Niles and Pease, 2003]. Moreover, we focus on the selectional restrictions of verbs w.r.t. the ontological type of their arguments, as well as their representation as necessary and sufficient conditions in the TBox. After a DL reasoner has identified the verb sense on the basis of these conditions, we make use of the more expressive Semantic Web Rule Language to calculate the inferences that are permitted on the selected interpretation.
\end{abstract}

\section{Introduction}

Verbs raise a number of challenges for computational linguistic applications, two of which will be addressed in this paper. Firstly, a lot of them are highly polysemous, which makes a careful disambiguation a prerequisite for the application of semantic web technologies. As an example, the same French verb encourager is normally translated by three different verbs in German, as illustrated in (1)-(3): 
(1) Un terroriste a encouragé 1 ma voisine à poser une bombe dans la cave. A terrorist has encouraged my neighbour to place a bomb in the basement. Ein Terrorist hat meine Nachbarin ermutigt, eine Bombe im Keller zu legen.

(2) La lettre a encouragé 2 ma voisine à poser une bombe dans la cave. The letter has encouraged my neighbour to place a bomb in the basement. Der Brief hat meine Nachbarin dazu bewegt, eine Bombe im Keller zu legen.

(3) Le gouvernement a encouragé 3 la recherche sur les armes biologiques. The government has encouraged research on biological weapons.

Die Regierung hat die Erforschung biologischer Waffen angeregt.

Note that (1) differs from (2) only by the ontological category of the subject, which is a human entity in (1) and a non-human ${ }^{1}$ one in (2). On the other hand, (3) differs from the previous two in that its object is a nonhuman entity (while the object denotes a human in (1) and (2)), and in that it does not take an infinitival complement. The second challenge concerns the computation and the weighting of the inferences triggered by verbs. The contrast between (1) and (2) offers a striking example: while the interpreter of (2) can take for granted that a bomb was placed, it can only be guessed that it was possible in (1). It is crucial to note that subtle differences like these do exist in French, which is further exemplified by the fact that (1) can be continued by the sentence ..., mais elle ne l'a pas fait. ('..., but she the neighbour] didn't do it.'), whereas (2) cannot (cf. Mari and Martin [2007]).

Following Bhatt [1999], let us call "actuality entailment" (AE) the entailment triggered by (2) - and to which the interpreter assigns the probability 1 - that an event satisfying the infinitival complement took place, and "weak inference" the one triggered by (1) - and to which the interpreter assigns a probability $p$ between 0 and 1 . Furthermore, we will say that when the AE is triggered, the verb instantiates its "implicative reading". ${ }^{2}$

Note that the presence of the non-human subject in (2) is only a necessary condition to trigger the $\mathrm{AE}$ (and consequently the implicative reading). For the AE to arise, the tense of the sentence has to be of a certain kind as well (i.e. a perfective tense, e.g. the passé composé in French). The interaction between lexical semantics and information pertaining to the textual level like tense and aspect must then be modelled appropriately to capture the facts.

\footnotetext{
${ }^{1}$ The fact that letters are generally written by humans is of no relevance here.

${ }^{2}$ The terminology is borrowed from [Karttunen, 1971]. Note however that verbs like encourager differ from what Karttunen originally calls "implicative verbs" (e.g. réussir à, 'manage to'), because the latter trigger an AE with any kind of tenses.
} 
A model which allows to identify and weigh appropriately the inferences triggered by verbs like encourager is highly desirable, since, first of all, verbs of the same class are pervasive in the lexicon and heavily present in official texts. ${ }^{3}$ Secondly, rating accurately the inference that an event described by a constituent took place is central for text understanding and summarisation.

As it is more convenient to present the implementation through a specific reading of a polysemous verb, we will firstly detail the lexical semantics of a specific interpretation of the verb encourager, namely the one translated by the German verb (zu etwas) bewegen and selected in (2).

In (1) and (2), encourager is a psychological verb in that it denotes an action or a state likely to trigger a certain psychological change of state of the entity denoted by the object $y$ (henceforth labelled "Experiencer"). This cannot be the case in (3), since a non-animate object (as research) cannot experience a psychological state or process. Rather, encourager denotes in (3) an action of the subject which is likely "to be good for the development of" the object. The evaluative component of this reading is the reason why we propose to call this reading the "axiological reading" of encourager.

Under the reading instantiated in (2), encourager is analysed here as meaning something like to be in a state with the goal that $y$ wants to $P$, or, if $y$ already has this desire, that $y$ wants to $P$ more than previously. $W$ ant $(s, d, t, P)$ describes the state of wanting $P$ at a degree $d$ at a time $t$. $P$ stands here for a three-place relation.

(4) encourager $_{2} \Rightarrow \lambda s \lambda y \lambda x \lambda P\left[\right.$ Theme $(s, x) \wedge \operatorname{Target}(s, y) \wedge \operatorname{Goal}\left(s, P_{2}\right) \wedge P_{2}=$ $\left[\lambda s_{1} \lambda d_{1} \lambda t \exists d_{2} \exists s_{2} \operatorname{Want}\left(s_{1}, d_{1}, P, t\right) \wedge \operatorname{Want}\left(s_{2}, d_{2}, P, t_{r}\right) \wedge t>t_{r} \wedge d_{2}>d_{1} \wedge\right.$ Experiencer $\left(s_{1}, y\right) \wedge$ Experiencer $\left.\left.\left(s_{2}, y\right)\right]\right]$

Plan of the paper. Section 2 discusses the model, focussing on selectional restrictions and formalisation of inference rules. Section 3 shows how these are applied to (2) in order to disambiguate the verb and its arguments, and to calculate the inferences based on this selection. We conclude in Section 4 with the advantages of our approach over existing lexical resources.

\section{Implementation of the Model}

In the following, we will describe how we model the inference triggers just mentioned as well as semantic representations like the one in (4) using OWL

\footnotetext{
${ }^{3}$ Examples of verbs displaying the alternation between (1) and (2) (thus triggering inferences of different strength in the two places) are autoriser $\grave{a} P$ 'to authorize to $P$ ', aider à $P$, 'to help to $P$ ', or exiger que $P$, 'to demand that $P$ '.
} 
DL and the more expressive Semantic Web Rule Language (SWRL). ${ }^{4}$

\subsection{Encoding of selectional argument restrictions}

As mentioned above, the primary triggers for selecting one sense over another is the presence (or absence) of syntactic arguments as well as their ontological type. For example in (2), the fact that (i) encourager subcategorises an infinitive, (ii) the subject is inanimate, and (iii) the direct object is animate, determine the sense of encourager in this sentence. To make this information available and processable, we use a straightforward encoding of these triggers as conditions on class definitions (see also Franconi [2003]), based on concepts of the Suggested Upper Merged Ontology ${ }^{5}$ (SUMO; Niles and Pease [2003]).

In particular, the different senses of a verb are modelled as subclasses of a general class that denotes an underspecified representation of the verb. However, the different verb senses do only subclass this generic representation in the lexicon, not in the concept hierarchy, since verb senses very frequently denote different concepts that are not subsumed by a common concept. In our concrete example, we assume 3 different senses of encourager in our lexicon, which differ w.r.t. to their axiomatic definition. (5) below shows the definition of the sense of encourager corresponding to the one in (2) above.

(5) encourager $2 \equiv$ encourager

$$
\begin{aligned}
& \exists \operatorname{obj}(\text { Agent } \sqcup \text { SocialRole }) \sqcap \forall \text { obj }(\text { Agent } \sqcup \text { SocialRole }) \\
& \exists \operatorname{subj}(\neg \text { Agent }) \sqcap \forall \operatorname{subj}(\neg \text { Agent }) \\
& \geq 1 \text { xcompPred owl:Thing }
\end{aligned}
$$

The first line of this definition states that encourager 2 is a subclass of the underspecified encourager. The subsequent line requires that there be values of the $o b j$ property which are either of type Agent or SocialRole, and further that all values of the $o b j$ property have to be within this set of Agents or SocialRoles. For capturing the inanimacy of the subject of encourager, the next line indicates that the values of the $s u b j$ property may not be of type Agent, and finally, the last line says that the xcompPred property (i.e. the main predicate of the embedded infinitive) has to have at least one value.

The general motivation for this encoding, which views the contextual triggers discussed above as necessary and sufficient conditions, is that a

\footnotetext{
${ }^{4}$ See http://www.w3.org/TR/owl-ref/ and http://www.w3.org/Submission/SWRL/.

${ }^{5}$ The reasons for using SUMO classes will become evident in Section 3.1 below.
} 
reasoner can infer - on the basis of a particular setting of contextual parameters (i.e. property values) - the specific type of an instance of the generic encourager. In the following, we will discuss the inference rules that are attached to each sense class, and which are evoked once a specific sense has been determined.

\section{$2.2 \quad$ Inference rules}

As was mentioned above, the different senses of encourager do not only differ w.r.t. to the necessary and sufficient conditions that are used to classify them, but also w.r.t. the inferences they allow. In our model, such inferences are encoded in the form of SWRL rules (see e.g. O'Connor et al. [2005]), as they require inference capacities which go beyond the scope of the inventory provided by OWL DL. The SWRL rule that corresponds to the semantic description of encourager 2 given in (4) is shown in Table 1 below, with the rule body in lines 1 to 8 and the rule head in lines 9 to 30 .

The first line represents the configuration in which the rule applies, i.e. an instance of encourager 2 with grammatical subject and object. Lines 2 to 8 make use of the SWRL extensions built-ins ${ }^{6}$ defined within the Protégé ontology editor [Knublauch et al., 2004] in order to create instances that are to be inserted into the representation, based on the analysis in (4). In lines 9 to 11, the grammatical subject is asserted as the theme of the state denoted by encourager, the grammatical object as target, and, as goal, the proposition that the degree of $y$ wanting to "place a bomb" is now greater than it was at a previous point in time. The two "wanting" states that are part of this proposition (lines 12 and 13) are described in lines 14 to 17 and 18 to 21 , where the "wanted" object corresponds to the embedded infinitive ${ }^{7}$. Lines 22 and 23 state that the previous degree of wanting is greater than the current one (note that the reference time $t r$ is not a variable), and that the grammatical object $y$ is the experiencer of both wanting states. Finally, lines 26 to 30 classify the remaining instances created in the rule body.

Although the rule appears to be quite specifically tailored to the particular use of encourager2, large parts of it can be re-used for senses of other verbs within this group of psychological verbs, while other parts can be parametrised to cover verbs of other groups. For example, the syntactic setting (cf. line 1) is applicable to any transitive verb, while the assignment

\footnotetext{
${ }^{6}$ See http://protege.cim3.net/cgi-bin/wiki.pl?SWRLExtensionsBuiltIns; the built-in function createOWLThing has been replaced with cOT in the table.

${ }^{7}$ Note that the four-place predicate $\operatorname{Want}(s, d, P, t)$ in (4) has been decomposed into a sequence of two-place predicates indicating the degree, time, and object of the "wanting".
} 


\begin{tabular}{|c|c|c|}
\hline 1 & syntactic configuration required for appli- & \multirow{9}{*}{ 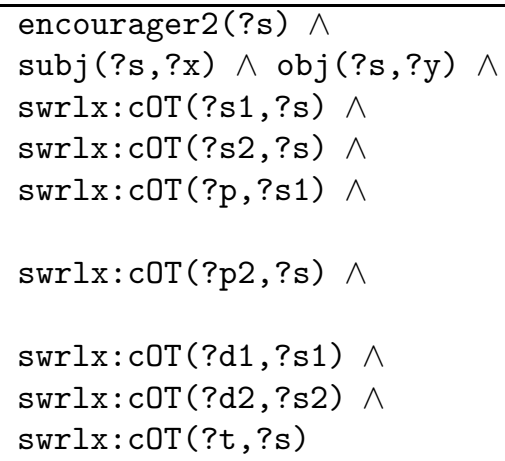 } \\
\hline & cation of rule & \\
\hline 2 & create previous state of wanting & \\
\hline 3 & create current state of wanting & \\
\hline 4 & $\begin{array}{l}\text { create proposition corresponding to em- } \\
\text { bedded infinitive }\end{array}$ & \\
\hline 5 & $\begin{array}{l}\text { create the proposition that is the goal of } \\
\text { encourager }\end{array}$ & \\
\hline 6 & create previous degree of wanting & \\
\hline 7 & create current degree of wanting & \\
\hline \multirow[t]{2}{*}{8} & create previous time & \\
\hline & & $\rightarrow$ \\
\hline 9 & $\begin{array}{l}\text { assert grammatical subject as theme of } \\
\text { encourager }\end{array}$ & theme (?s,?x) $\wedge$ \\
\hline 0 & $\begin{array}{l}\text { assert grammatical object as target of } \\
\text { encourager }\end{array}$ & target $(? \mathrm{~s}, ? \mathrm{y}) \wedge$ \\
\hline 1 & assert proposition as goal of encourager & goal (?s,?p2) ^ \\
\hline & $\begin{array}{l}\text { assert that previous state is part of the } \\
\text { proposition }\end{array}$ & hasPart (?p2,?s1) $\wedge$ \\
\hline & $\begin{array}{l}\text { assert that current state is part of the } \\
\text { proposition }\end{array}$ & hasPart (?p2,?s2) $\wedge$ \\
\hline 4 & assert previous state of wanting & WANTING(?s1) $\wedge$ \\
\hline 5 & assert previous degree of wanting & degree $(? \mathrm{~s} 1, ? \mathrm{~d} 1) \wedge$ \\
\hline 16 & assert object of wanting & object $(? \mathrm{~s} 1, ? \mathrm{p}) \wedge$ \\
\hline 7 & assert time of previous wanting & time $(? \mathrm{~s} 1, ? \mathrm{t}) \wedge$ \\
\hline .0 & assert current state of wanting & WANTING(?s2) $\wedge$ \\
\hline & assert current degree of wanting & degree $(? \mathrm{~s} 2, ? \mathrm{~d} 2) \wedge$ \\
\hline & assert object of wanting & object $(? s 2, ? p) \wedge$ \\
\hline & $\begin{array}{l}\text { assert reference time as time current of } \\
\text { wanting }\end{array}$ & time $(? \mathrm{~s} 2, \mathrm{tr}) \wedge$ \\
\hline & $\begin{array}{l}\text { assert that time of previous wanting is be- } \\
\text { fore reference time }\end{array}$ & before $(? t, t r) \wedge$ \\
\hline & $\begin{array}{l}\text { assert that current degree of wanting is } \\
\text { greater than previous }\end{array}$ & greaterThan $(? \mathrm{~d} 2, ? \mathrm{~d} 1) \wedge$ \\
\hline & $\begin{array}{l}\text { assert grammatical object as experiencer of } \\
\text { previous wanting }\end{array}$ & experiencer(?s1,?y) $\wedge$ \\
\hline & $\begin{array}{l}\text { assert grammatical object as experiencer of } \\
\text { current wanting }\end{array}$ & experiencer(?s2,?y) $\wedge$ \\
\hline & assert proposition & Proposition(?p) $\wedge$ \\
\hline 1 & assert proposition & Proposition(?p2) $\wedge$ \\
\hline ic & assert degree & Degree $(? d 1) \wedge$ \\
\hline & assert degree & Degree $(? d 2) \wedge$ \\
\hline 50 & assert time & Time (?t) \\
\hline
\end{tabular}

Table 1: SWRL rule implementing the description of encourager 2 in (4) 
of semantic roles (lines 9 to 11), the specification of the involved states (or events) in lines 14 to 21 and 24 to 25 , as well as the more specific temporal and degree assertions in lines 22 to 23 can be parametrised to suit other verb senses. For instance, the semantics of verbs like inviter à $P$ ('to invite to $P^{\prime}$ ), inciter à $P$ ('to incite to $P^{\prime}$ '), pousser $\grave{a} P$ ('push to $P^{\prime}$ ') and others also involve a comparison between different degrees of wanting $P$ associated to different states. One possibility to achieve parametrisation is to store information on these individual segments as property values for the sense classes. These are read out by an external application and re-inserted into the model in the form of a SWRL rule. This way, it is not necessary to store inference rules for every single word sense, but to have only a small set of generally applicable static rules in the model, while specific ones applying in a given discourse are created dynamically using externally completed rule templates.

\section{Disambiguation and Calculation of Inferences}

In order to select the correct reading of a verbal predicate in a sentence like (2) and, moreover, to generate the appropriate semantic representation on the basis of this choice, our system passes a number of distinct analysis steps. Basically, the system receives input from a syntactic parser and tries to determine the correct senses of both the verbal predicate and its syntactic arguments, before calculating the inferences permitted on this interpretation. The process is summarised in Figure 1.

For the scope of this paper, we will ignore details on the syntactic analysis that precedes the semantic processing steps, and instead assume a syntactic parser which returns output like the one depicted in Figure 1, providing information on the predicate (encourager), its syntactic arguments (lettre, voisin and the infinitival complement), its modal context (e.g. embedding under pouvoir, 'can, be able to'), and the tense in which the predicate is used. These context features are crucial for determining the inferences that may be drawn, and thus play an important role in the semantic processing steps which build on the syntactic analysis (see below).

\subsection{Disambiguation of the predicate and its arguments}

Before the correct sense of the verbal predicate can be selected, its syntactic arguments have to be disambiguated first. In order to achieve this, we apply a methodology that has been presented in [Spohr, 2008], which is very much in line with standard approaches to word-sense disambiguation (see e.g. 


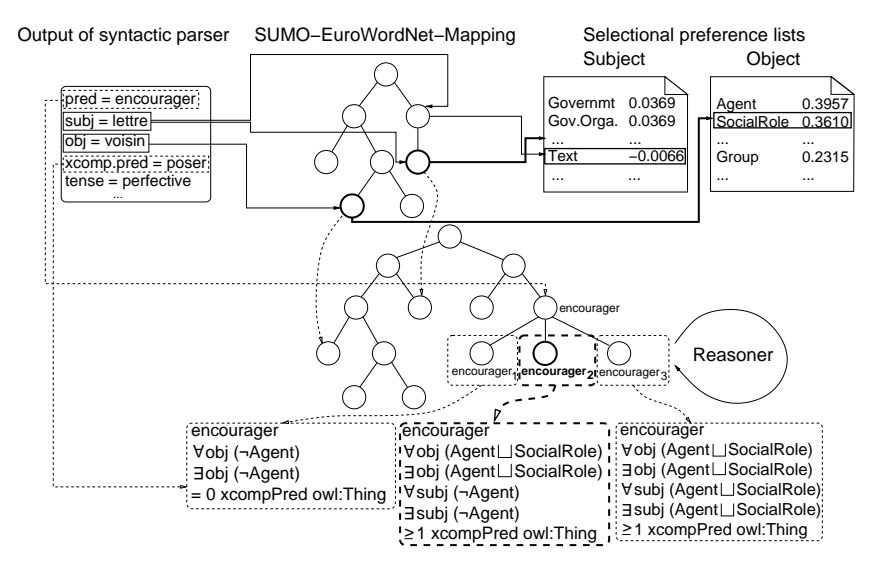

Figure 1: Schema of the process of determining the intended sense of encourager in (2) from syntactically parsed input

[Schulte im Walde, 2008] for a recent account of related work in this field). The major difference is that it makes use of ontological categories based on a mapping between the French EuroWordNet - a lexical semantic resource for French (EWN; see e.g. Vossen [1998]) - and the Suggested Upper Merged Ontology (SUMO; Niles and Pease [2003]). By applying this methodology to a verb like encourager, we obtain lists of selectional preferences w.r.t. to the ontological types of its subject and object (see top righthand corner of Figure 1). For the actual disambiguation, the different senses of the subject (lettre in the present case) are looked up in the SUMO-EWN mapping, and the sense scoring highest in the corresponding selectional preference list is selected. The words are then asserted as instances of the respective class (in this example Text for lettre and SocialRole for voisin) in the model.

The disambiguation of the arguments is not a deterministic process. However, when viewed from the abstract level of SUMO concepts, the senses distinguished in EWN are often still closely related so that their distinctions have no impact on the interpretation of the verb and thus the selection of the appropriate sense. E.g., although the three senses of lettre in EWN map onto two SUMO concepts (Text and Character), they are still subsumed under the common class ContentBearingPhysical, which suffices to select the correct sense of encourager irrespective of the particular sense of lettre. Thus, even though arguments may be disambiguated towards the wrong sense, the interpretation of the verb stays the same and the inferences drawn on the basis of this selection remain unaffected. Once the 
arguments have been disambiguated, they are linked to the instance representing encourager. The intermediate ABox obtained from the operations so far looks as follows.

(6) encourager $\left(\_s\right) \wedge \operatorname{subj}\left(\_s\right.$, lettre $) \wedge \operatorname{obj}\left(\_s\right.$, voisin $) \wedge \operatorname{Text}($ lettre $) \wedge$ SocialRole(voisin)

The next step consists in determining the correct sense of encourager. As mentioned in Section 2 above, selectional restrictions have been implemented as necessary and sufficient conditions on class definitions, which allows a reasoner to infer the type of the instance on the basis of these conditions. With the configuration in (6), the reasoner ${ }^{8}$ correctly infers the instance of encourager as being of the more specific type encourager 2 , as this is the only class satisfying the condition of having a subject that is not an Agent, while having an object either of type Agent or SocialRole (cf. Figure 1).

\subsection{Calculation of inferences}

The assertion of encourager2(_s) in combination with subj(_s, lettre $) \wedge$ obj(_s, voisin) causes the SWRL rule in Table 1 above to fire, so that the inferences can be calculated and inserted into the ABox. For this task we used version 7 of the Jess rule engine ${ }^{9}$. The result of the rule application is illustrated in Figure 2 and represents all assertions made on the basis of the input sentence (2). ${ }^{10}$ The logical form of the graph is given below.

(7) encourager $2\left(\_s\right) \wedge$ theme $\left(\_s\right.$, lettre $) \wedge \operatorname{target}\left(\_s, \operatorname{voisin}\right) \wedge$ goal(_s, _p2)

$\wedge$ hasPart $\left(\_p 2, \_s 1\right) \wedge W A N T I N G\left(\_s 1\right) \wedge$ degree $\left(\_s 1, \_d 1\right) \wedge$ object $\left(\_s 1, \_p\right)$

$\wedge$ time $\left(\_s 1, \_t\right) \wedge h a s P a r t\left(\_p 2, \_s 2\right) \wedge W A N T I N G\left(\_s 2\right) \wedge$ degree(_s2,_d2)

$\wedge$ object $\left(\_s 2, \_p\right) \wedge$ time $\left(\_s 2, \operatorname{tr}\right) \wedge$ before $\left(\_t, t r\right) \wedge$ greaterThan $\left(\_d 2, \_d 1\right)$

$\wedge$ experiencer $\left(\_s 1\right.$, voisin $) \wedge$ experiencer $\left(\_s 2\right.$, voisin $) \wedge \operatorname{Proposition}\left(\_p\right)$

$\wedge$ Proposition $\left(\_p 2\right) \wedge$ Degree $\left(\_d 1\right) \wedge$ Degree $\left(\_d 2\right) \wedge$ Time $\left(\_t\right)$

After the application of this rule, we can execute the rule corresponding to the representation of the embedded infinitive, which is triggered by the use of the perfective tense in sentence (2). In order to avoid repetition, we will restrict ourselves to presenting only those statements that are introduced

\footnotetext{
${ }^{8}$ We have used the Pellet OWL DL reasoner (http://pellet.owldl.com/).

${ }^{9}$ See http://www. jessrules.com/.

${ }^{10}$ The visualisation of the graph has been done using the Protégé Jambalaya plug-in (see http://www.thechiselgroup.org/jambalaya).
} 


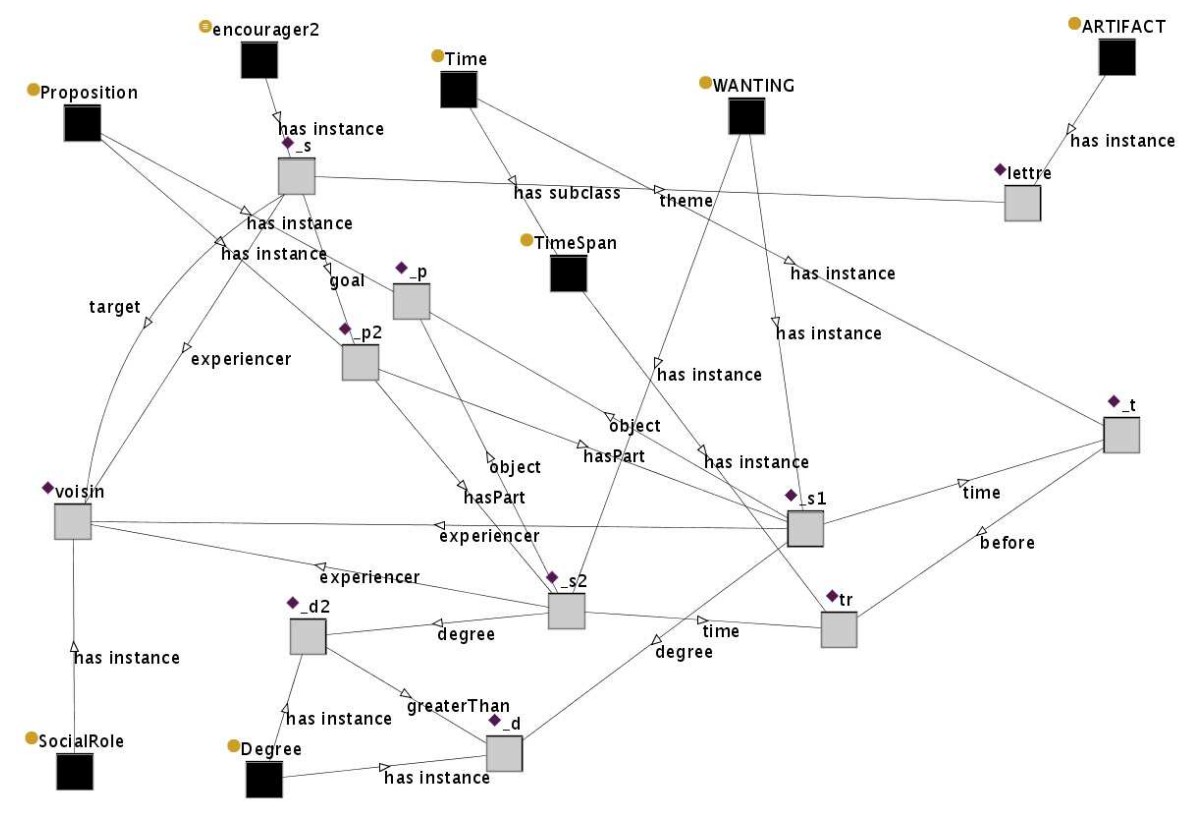

Figure 2: Model after execution of the SWRL rule in Table 1

by the embedded infinitive. Again, a certain sense of poser is instantiated in the lexicon, which then triggers the application of an inference rule ${ }^{11}$.

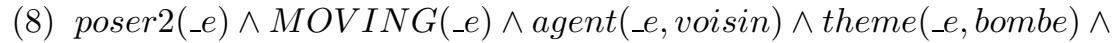
Weapon $($ bombe $) \wedge$ inside $\left(\_s 3\right.$, cave $) \wedge \operatorname{Artifact}($ cave $) \wedge$ theme $\left(\_s 3\right.$, bombe $) \wedge$ outside $\left(\_s 4\right.$, cave $) \wedge$ theme $\left(\_s 4\right.$, bombe $) \wedge$ time $\left(\_s 4, \_t 2\right) \wedge$ time $\left(\_e, \_t 3\right) \wedge$ before $\left(\_t 2, \_t 3\right) \wedge$ cause (_e, _s3)

The interpretation of this logical form is that a "moving" event _ $e$ took place that causes the bombe, which was outside of the basement at a previous state $\_s 4$, to be inside the basement at a state _s3. Moreover, this event _e is linked to the representation given in (7) as being a part of the proposition _ $p$. As a result, the model captures appropriately the fact that in sentence (2), the verb encourager instantiates what we called its implicative reading.

\footnotetext{
${ }^{11}$ Weapon and Artifact are SUMO concepts subsuming the senses of bombe and cave.
} 


\section{Conclusion}

We have presented an approach to modelling polysemous verbs using standard formalisms such as OWL and SWRL. We have shown how the disambiguation of the verbs and their arguments can be performed in this setting, and how inferences can be calculated and inserted into a representation that can be interpreted by tools developed in the context of the Semantic Web.

One of the advantages of our approach is that fine-grained sense distinctions based on contextual features enable accurate annotation of particular senses, and with it the calculation of inferences allowed by the respective sense. Moreover, the resource combines formal semantics with up-to-date technology for semantic processing and is thus more formalised and detailed than existing lexical semantic resources. WordNet [Fellbaum, 1998], for example, distinguishes three senses of to encourage (1. 'support, back up', 2. 'inspire, animate...', 3. 'induce, stimulate...'), without accounting for the difference between implicative and non-implicative readings: the examples show clearly that both are subsumed by sense 3 (e.g. 'The ads induced me to buy a VCR' vs. 'My children finally got me to buy a computer'). FrameNet [Baker et al., 1998] contains only one sense of to encourage, which it associates with the AtTEMPT_SuAsion frame. This frame corresponds to the non-implicative reading (definition: 'The Speaker expresses through language his wish to get the Addressee to act. There is no implication that the Addressee forms an intention to act, let alone acts.'), whereas e.g. SuASION implies that the Addressee forms an intention or accepts some content. However, encourage is neither associated with this latter frame, nor with a frame implying an action. Moreover, it does not emphasise the contextual parameters mentioned above (e.g. the presence of arguments and their ontological type), which trigger certain interpretations and are crucial for determining the sense of a verb as well as its inferences. More closely related is VerbNet [Kipper Schuler, 2005], though the major difference lies in the formal semantic description that is the output of our analysis.

Although the system is - due to lack of coverage - not in a state of being applied to sophisticated reasoning tasks such as the RTE challenge [Dagan et al., 2005], the inclusion of the contained knowledge into existing systems designed for such tasks seems very promising. The RTE challenge consists in determining, given two text fragments, whether one text fragment is entailed by the other. In our examples, the hypothesis "A bomb has been placed." can only be inferred from sentence (2), not from (1). This shows that great detail in the semantic description is a definite asset and an important step beyond the information contained in existing lexical semantic resources. 


\section{Acknowledgements}

This work has been done within the project 'Polysemy in a Conceptual System' (SFB 732, project B5), funded by the German Research Foundation.

\section{References}

Collin F. Baker, Charles J. Fillmore, and John B. Lowe. The Berkeley FrameNet project. In Proceedings of COLING/ACL, Montreal, 1998.

Rajesh Bhatt. Covert Modality in Non-Finite Contexts. PhD thesis, University of Pennsylvania, 1999.

Ido Dagan, Oren Glickman, and Bernardo Magnini. The PASCAL Recognising Textual Entailment Challenge. In Proceedings of the PASCAL Challenges Workshop on RTE, Southampton, UK, 2005.

Christiane Fellbaum, editor. WordNet - An Electronic Lexical Database. MIT Press, Cambridge, MA, USA, 1998.

Enrico Franconi. Natural Language Processing. In The Description Logic Handbook: Theory, Implementation and Applications. CUP, 2003.

Lauri Karttunen. Implicative verbs. Language, 47:340-358, 1971.

Karin Kipper Schuler. VerbNet: A Broad-Coverage, Comprehensive Verb Lexicon. PhD thesis, University of Pennsylvania, 2005.

Holger Knublauch, Mark A. Musen, and Alan L. Rector. Editing description logic ontologies with the Protégé OWL plugin. In Proceedings of DL 2004, Whistler, BC, 2004.

Alda Mari and Fabienne Martin. Tense, abilities and actuality entailment. In Maria Aloni, Paul Dekker, and Floris Roelofsen, editors, Proceedings of the Sixteenth Amsterdam Colloquium, pages 151-156, 2007.

Ian Niles and Adam Pease. Linking Lexicons and Ontologies: Mapping WordNet to the Suggested Upper Merged Ontology. In Proceedings of IKE '03, Las Vegas, NV, 2003.

Martin O'Connor, Holger Knublauch, Samson Tu, Benjamin Grosof, Mike Dean, William Grosso, and Mark Musen. Supporting Rule System Interoperability on the Semantic Web with SWRL. In Proceedings of the 4th International Semantic Web Conference, Galway, Ireland, 2005. 
Sabine Schulte im Walde. The Induction of Verb Frames and Verb Classes from Corpora. In Corpus Linguistics. An International Handbook, volume 29.1 of Handbooks of Linguistics and Communication Science, pages 952971. Mouton de Gruyter, Berlin, 2008.

Dennis Spohr. Extraction of Selectional Preferences for French using a Mapping from EuroWordNet to the Suggested Upper Merged Ontology. In Proceedings of the 4th Global WordNet Conference, Szeged, Hungary, 2008.

Piek Vossen, editor. EuroWordNet: A Multilingual Database with Lexical Semantic Networks. Kluwer Academic Publishers, 1998. 